\title{
Acute Disseminated Encephalomyelitis: A Rare Neurological Complication of Mixed Malaria
}

Authors

\section{V.Kotrashetti, Kapil Bainade, Mumtaz Shariff, Vijay Baburao Sonawane, Imran Patel}

\begin{abstract}
Acute disseminated encephalomyelitis (ADEM) is an initial inflammatory demyelinating event with multifocal neurological deficits, typically accompanied by encephalopathy ${ }^{1}$. Generally, the diagnosis of ADEM is made by clinical history, neurological manifestations and magnetic resonance images (MRI) of the central nervous system. ADEM has not been recognized as a common neurological complication of malaria. As per the Pubmed search only 4 cases of ADEM as a neurological complication of malaria has been reported.

We report a 8 year old male child with cerebral malaria and ADEM which is a rare neurological complication and diagnosis was made on the basis of MRI Brain.

Keywords: Acute Disseminated Encephalomyelitis (ADEM); Cerebral Malaria; MRI
\end{abstract}

\section{Case Report}

An 8 year male child admitted with complaints of high grade fever and altered sensorium for one day. On admission, child was sick looking with Glasgow coma scale (GCS) 10/15, temperature $38.5^{\circ} \mathbf{C}$, pulse rate $120 / \mathrm{min}$, respiratory rate $34 / \mathrm{min}$ and blood pressure 110/70 $\mathrm{mm}$ of $\mathrm{Hg}$. General physical examination revealed pallor and icterus. Abdominal examination showed hepatomegaly. On CNS examination, the child was drowsy with hypertonia and positive Babinski's response and no signs of meningitis.

Laboratory investigations showed $\mathrm{Hb} 5 \mathrm{~g} / \mathrm{dl}$, white blood cells (WBC) $7 \times 103 / \mu$ land platelets $19 \times 103 / \mu 1$. Serum bilirubin $7.4 \mathrm{mg} / \mathrm{dl}$ and blood sugar was normal. Rapid malaria antigen test was positive for P. vivax and P.falciparum.

Treatment was initiated with Injection Artesunate $2.4 \mathrm{mg} / \mathrm{kg} / \mathrm{dose}$ repeated after 12 and $24 \mathrm{~h}$ as per guidelines of the National Vector Borne Disease Control Programme, India for treatment of severe malaria. Packed cells and platelets transfusion were given. Child became afebrile after $24 \mathrm{hrs}$ but sensorium did not improve even after $72 \mathrm{hrs}$.

MRI T2 flair shows patchy areas of restricted diffusion involving bilateral periventricular and subcortical white matter, splenium, genu and body of corpus callosum suggestive of acute disseminated encephalomyelitis (ADEM) (FIG. A,B.C.D). The condition of the child improved on $5^{\text {th }}$ day of illness with antimalarial and supporting management. No steroids were given. 


\section{JMSCR Vol.||03||Issue||08||Page 7118-7120||August 2015}
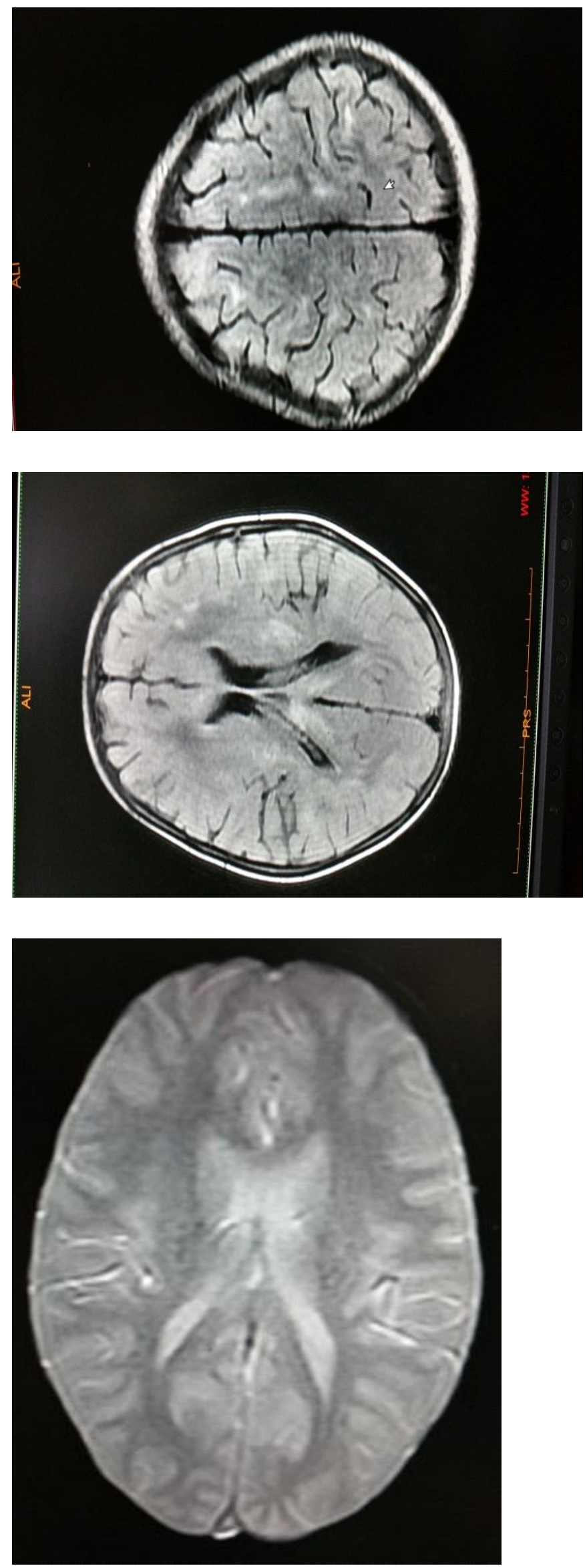

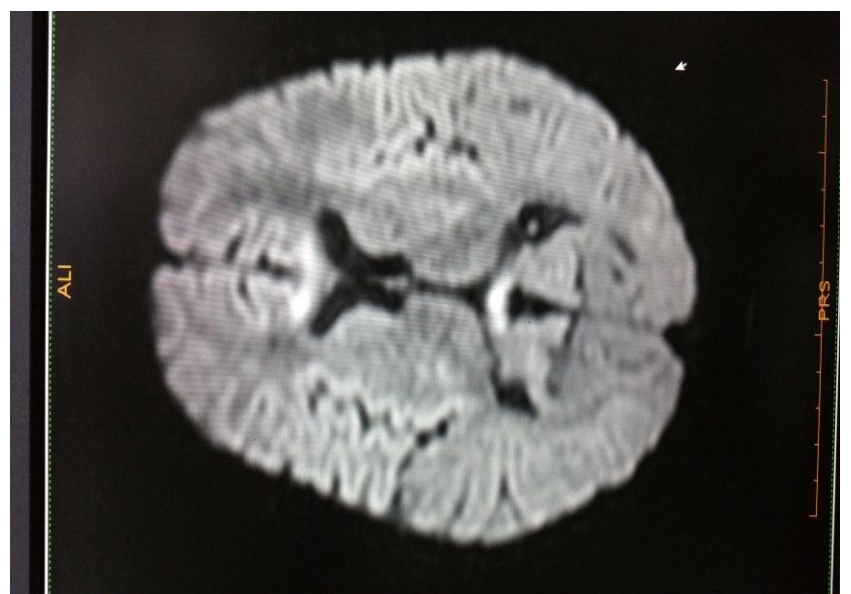

Fig.(A,B)MRI flair sequences showing hyperintense lesions in subcortical and bilateral parietal cortex (arrows)

(C) Axial T2 weighed image showing bilateral symmetrical altered signal intensity involving subcortical white matter

(D) DWI shows restriction in corpus callosum genu and splenium.

\section{Discussion}

Cerebral malaria is severe $P$. falciparum malaria with cerebral manifestations, usually including coma (Glasgow coma scale $<11$, Blantyre coma scale $<3$ ) or malaria with coma persisting for $>30$ min after a seizure ${ }^{2}$. They usually recovers within 72hours of treatment. The sequestration of infected erythrocytes (IEs) in cerebral microvascular beds is the basic pathology in cerebral malaria. The precise mechanisms involved in the onset of neuropathology remain unknown, but parasite sequestration in the brain, metabolic disturbances and immune response plays a major role.

Acute disseminated encephalomyelitis (ADEM) is classically described as a uniphasic syndrome occurring in association with an vaccination or systemic viral infection. Pathologically there is perivascular inflammation, edema, and demyelination within the central nervous system. Magnetic resonance imaging (MRI) is important in establishing the diagnosis. Findings may progress over a relatively short period of time, consistent with evolution of the disease process. MRI abnormalities vary in location. Lesions are typically bilateral but asymmetric and tend to be 
poorly marginated. Almost all patients have multiple characteristic lesions of demyelination in the deep and subcortical white matter. The thalami and basal ganglia are frequently affected. Our child haslesions predominantly in subcortical and bilateral parietal cortex.

ADEM has been reported following a viral infection and vaccination, rarely it is reported following parasitic infection like malaria as case reported by Sharmaet $\mathrm{al}^{3}$ and Amit agrawal et $\mathrm{al}^{4}$ similar to our case.

\section{References}

1. Nelson. PEDIATRÍA. 19.AEdición.

Volume n 1. Robert M. Kliegman, MD.

Professor And ... Bonita F. Stanton, MD

... Nina F. Schor, MD, Phd ... Nelson

Textbook

Of

Pediatrics ..... 593.3 Encefalomielitis

Diseminada Aguda (EMDA).

2. Apps.Who.Int/Iris/Bitstream/10665/16244

1/1/9789241549127_Eng.Pdf..By World

Health Organization - 2015 WHO

Guidelines For The Treatment Of

Malaria-Second Edition.

3. Sharma N, Varma S, Bhalla A. Acute

Disseminated Encephalomyelitis After

Treatment Of Severe Falciparum

Malaria. Indian J Med Sci 2008;62:69-70 .

4. Amit Agarwal, SwetaGoyal et al. Acute Demyelinating Encephalomyelitis In A Child Following Malaria. Indian Pediatrics, Current Issue: November 2012;

Volume 49: Number 11. 